\title{
Otimizando o Uso de Sistemas de Telecooperaçâo - Uma Abordagem dos Aspectos Humanos
}

\author{
Ana Paula Freitas Mundim, Msc. \\ Doutoranda do Dep. Engenharia Mecânica \\ Escola de Engenharia de São Carlos - USP \\ Av. Dr. Carlos Botelho, 1465 \\ São Carlos - SP 13560580 - Brasil \\ Amundim@sc.usp.br \\ Luciane Meneguin Ortega, Msc. \\ Doutoranda do Dep. Engenharia Mecânica \\ Escola de Engenharia de São Carlos - USP \\ Av. Dr. Carlos Botelho, 1465 \\ São Carlos - SP 13560580 - Brasil \\ lmortega@sc.usp.br \\ Prof. Dr. Eduardo Vila \\ Professor do Dep. Engenharia Mecânica \\ Escola de Engenharia de São Carlos - USP \\ Av. Dr. Carlos Botelho, 1465 \\ São Carlos - SP 13560580 - Brasil \\ Evila@sc.usp.br
}

\section{Resumo}

Devido a competiçāo internacional, as empresas de manufatura estāo investigando novos processos de integraçāo visando manter suas posiçōes competitivas. A implementação de sistemas de telecooperação representa uma abordagem direcionada pela combinação de modernas tecnologias de informação e pela integração de processos cooperativos. Entretanto, para se alcançar os benefícios que o uso dos sistemas de telecooperação podem proporcionar, as empresas necessitam organizar suas equipes de trabalho, segundo alguns critérios. O objetivo deste artigo é descrever nāo só a funcionalidade destes sistemas no contexto da manufatura, mas também apresentar os principais critérios para a formaçāo de eficientes equipes de trabalho a distância. $\mathrm{O}$ artigo ainda apresenta dois casos práticos ilustrando a importância destes critérios.

Palavras-chave: telecooperação, sistemas colaborativos, trabalho em grupo e cultura.

\begin{abstract}
In view of increased international competition, manufacturing enterprises are investigating new integrating processes to maintain their competitive position. The set-up of telecooperation systems represents an approach towards a combination of modern communication and information technologies and integration of co-operative processes. However, to make profit of the benefits that the use of the telecooperation systems can proportionate, enterprises need to organise their workgroups, according to some criteria. The aim of this paper is not only to describe the functionality of these systems and their role in the manufacture context, but also to present the main criteria to form efficient distributed workgroups. The paper also presents two practical cases illustrating the importance of the criteria described.
\end{abstract}

Keywords: telecooperation, collaborative systems, groupwork and culture. 


\section{Abordagem}

Novas tecnologias de informação e comunicação permitem hoje que um grande número de pessoas $\mathrm{e}$ organizações manipulem (aquisição, armazenamento e processamento) uma crescente quantidade de informações. Ao invés de realizar tudo internamente a cooperação, através do compartilhamento de informações, pode ser muito rentável para as empresas (Picot et al., 1997). Outro fator que vem sendo alterado é a forma de coordenação das atividades econômicas, passando de uma forma hierárquica para uma forma orientada ao mercado.

As empresas estão se tornando organizações sem fronteiras, configuradas em menores e mais autônomas unidades organizacionais, com um alto grau de interação entre estas unidades, dentro ou fora da organização. O objetivo é adquirir um alto grau de agilidade que pode ser definido como a habilidade de uma organização em responder eficientemente a eventos inesperados (Preis et al., 1996). Uma conseqüência principal é a estratégia empresarial de concentrar-se em suas competências essenciais (Hamuel \& Prahalad, 1994). O foco nas competências essenciais leva as empresas a terem de cooperar para oferecer produtos e serviços completos ao mercado consumidor.

Este cenário permite uma atuação dinâmica e global de diferentes atores, simultaneamente, dentro de um setor de negócios (clientes, competidores, fornecedores. Deste modo, o desenvolvimento de uma eficiente infra-estrutura de informação que permita a uma empresa atuar e responder à esta dinamicidade do negócio, onde distâncias físicas não se apresentam mais como um obstáculo, pode ser considerada uma importante vantagem competitiva.

Considerando espaço e tempo, uma eficiente tecnologia de informação pode unir processos que antes encontravam-se descentralizados e distribuídos. $\mathrm{Na}$ organização de processos de negócios, a abordagem de processamento paralelo de estruturas seqüenciais vai ser substituída por um meio de processamento de estruturas, de maneira a melhorar a harmonização e supostamente, acelerar a conclusão do processo (Eversheim et al.,1997). A expansão de cooperação além das zonas de fuso horário permitem ininterrupta exploração de recursos e experiências, prometendo um novo ritmo ao desenvolvimento mundial. Em contraste com a estratégia de integração vertical, a integração virtual das funções de uma corporação permite que as estruturas dentro das empresas se tornem mais flexíveis devido a hierarquias mais achatadas e a uma redução no montante de capital requerido.

Este artigo descreve o uso dos sistemas de telecooperação para suporte à processos cooperativos na manufatura, seja entre empresas ou dentro delas mesmo. Primeiramente, uma definição dos sistemas é apresentada, assim como suas principais ferramentas. Em seguida, descreve-se a quais processos os sistemas se adequam melhor. Entretanto, para se alcançar os benefícios que o uso dos sistemas de telecooperação podem proporcionar, as empresas necessitam organizar suas equipes de trabalho, segundo alguns critérios. De acordo com esta premissa são apresentados os principais critérios para a formação de eficientes equipes de trabalho a distância. Por fim, casos práticos são descritos a fim de ilustrar a importância destes critérios.

\section{Tecnologias de Trabalho em Grupo}

No ambiente atual de negócio que enfrenta a competitividade global demandando das empresas, desenvolvimentos de produtos de alta qualidade, cada vez mais rápido e com menor custo, reconheceu-se a necessidade de desenvolver aplicações que possibilitem o trabalho e a produção em conjunto.

Os sistemas de telecooperação ou sistemas de trabalho em grupo suportados por computador objetivam prover suporte computacional às pessoas para a realização de uma determinada atividade em conjunto. Eles permitem não só que pessoas dentro de uma mesma empresa possam trocar informações rapidamente, como também permitem a interação entre diferentes organizações, 
eliminando distâncias, aproximando pessoas, reduzindo tempo e custos e aumentando a produtividade (Mundim, 1999).

Neste panorama, os desenvolvedores de softwares estão visualizando rapidamente o potencial para um novo mercado de softwares especializados (Groupware). Os provedores de redes estão aperfeiçoando a conectividade e a alta largura de banda requerida pelos sistemas de telecooperação. $\mathrm{O}$ que vem atraindo a atenção das organizações, pois em um ambiente mutável, muito se dependerá da eficiência dos grupos de trabalho.

Sistemas Groupware costumam ser definidos como tecnologia de informação usada para ajudar pessoas trabalharem juntas mais eficientemente (Ellis et al., 1991). Os benefícios genéricos de Groupware são: melhora no fluxo de trabalho, mudança de tempo e locação, melhoria na comunicação através dos meios computacionais, organização, indexação, armazenamento e procura de informações e compartilhamento de recursos e de hardware (Wood, 1993). Entretanto, para implementar estes ganhos por meio de um ambiente cooperativo suportado por computador (CSCW Computer-Supported Co-operative Work) torna-se primordial a existência de um software de colaboração.

$\mathrm{O}$ termo $\mathrm{CSCW}$ pode ser definido como a disciplina de pesquisa para o estudo das técnicas e metodologias de trabalho em grupo e das formas como a tecnologia pode auxiliar este trabalho (Wilson, 1991). Ou seja, os termos CSCW e sistemas Groupware são termos usados para descrever esta tecnologia de suporte ao trabalho em grupo através do computador. No entanto, Groupware tende a ser mais orientado tecnologicamente, enquanto CSCW prefere levar em total consideração as características do comportamento humano no design e na implementação de suportes computacionais para o processo de trabalho em grupo [GRU94]. Ou seja, CSCW possui características mais abrangentes que os sistemas Groupware, pois além de considerar os aspectos tecnológicos dos sistemas computacionais, engloba também os aspectos humanos requeridos e desejáveis para a implementação e a eficiente utilização destes sistemas.
Segundo GRUDIN (1994), uma forma de classificar os sistemas Groupware é segundo o nível de funcionalidade da aplicação do sistema. Neste tipo de classificação procura-se estabelecer tipos de aplicações Groupware. Tem-se então:

- sistemas de mensagem, destinados a suportar trocas assíncronas de mensagens (exemplo: e-mail);

- sistemas de editoração multi-usuários, que procura apoiar o trabalho conjunto e simultâneo de duas ou mais pessoas em um mesmo texto ou gráfico;

- sistemas de apoio à decisão e salas de encontro eletrônico, que procuram melhorar a produtividade dos processo de tomada de decisão e a qualidade das mesmas;

- conferência via computador, aqui os sistemas computacionais servem como meio de comunicação entre grupos de pessoas, ressaltando a convergência entre as telecomunicações e a computação;

- sistemas para coordenação de grupos de pessoas que apoiam a coordenação de grupos de pessoas trabalhando em tarefas comuns.

Os sistemas de telecooperação encaixam-se na classificação de conferência via computador, englobando os sistemas de mensagens e sistemas de editoração multiusuários, que envolvem diferentes componentes, como: áudio, vídeo, dados e compartilhamento de aplicação (Figura 1). Mais ainda, a telecooperação baseia-se no $\mathrm{CSCW}$, que envolve pesquisas de design e implementação de conexões entre pessoas, tanto como, de acesso a informações.

$\mathrm{CSCW}$ começou com o compartilhamento entre tecnólogos, economistas, psicólogos, educadores e antropologistas de experiências, possibilidades técnicas e restrições para estudo e análise de sistemas e ambientes que suportem o trabalho das pessoas em grupo e ou em organizações. Ou seja, este ramo busca estudar como as pessoas trabalham e desenvolver técnicas que facilitem a construção de softwares para trabalhos em equipe (ELLIS et al., 1991). Por isso, o CSCW envolve áreas tais como: Sistemas Distribuídos, Comunicação, Interação Homem Máquina, Inteligência Artificial e Ciências Sociais. 


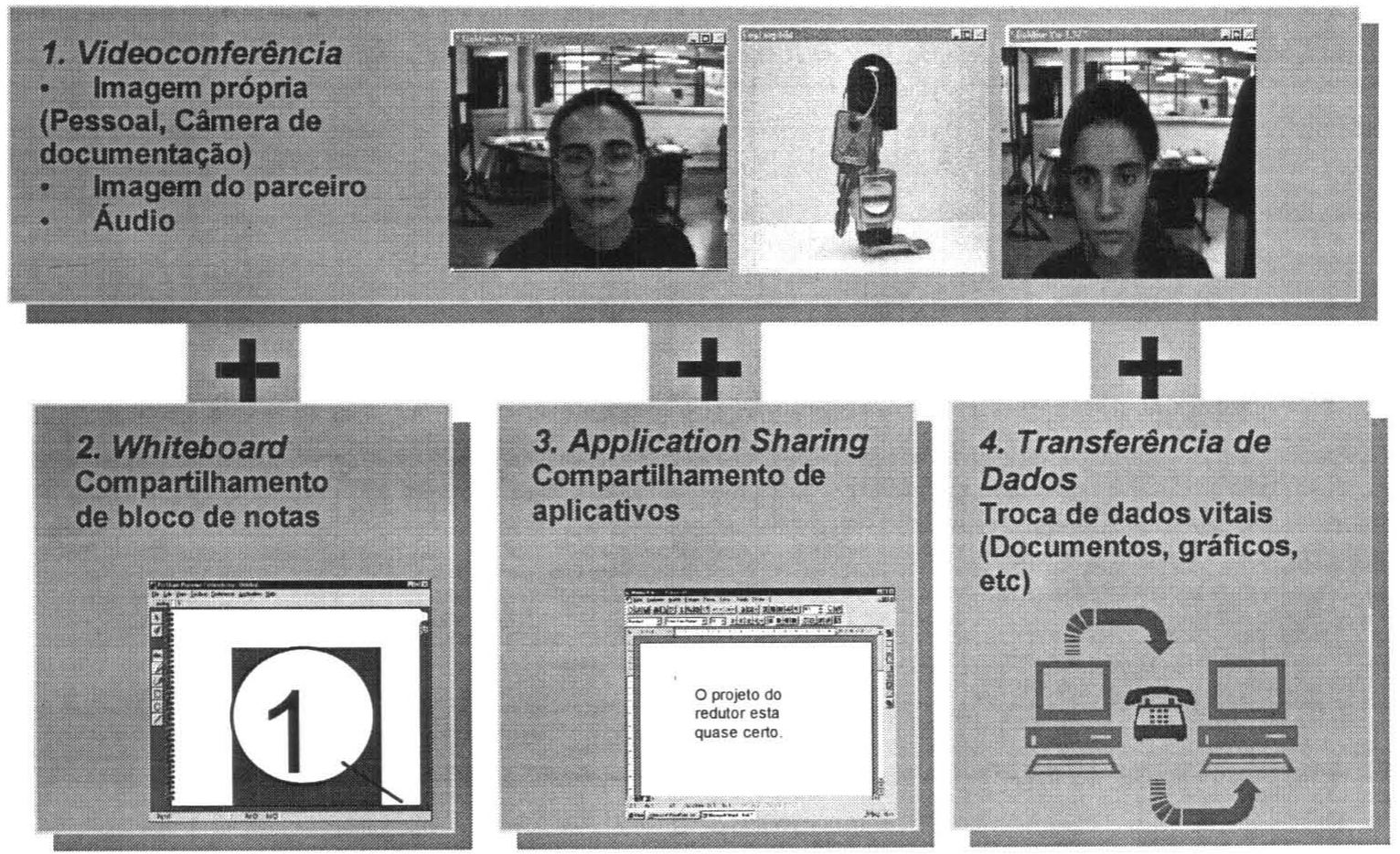

Figura 1 - Componentes de um Sistema de Telecooperação

A sociedade adquire muito de suas características através do modo como as pessoas se interagem. Como as tecnologias de informação e outras formas de comunicação eletrônica continuam desenvolvendo-se, surgirão novos e diferentes meios de interação entre as pessoas. Um provável resultado deste "casamento" tecnológico será um vasto sistema de organização que integrará processamento de informações e atividades de comunicação. O estudo de tais sistemas é o foco do CSCW.

Alguns exemplos de sistemas CSCW são: "Computer Aided Design" (CAD), salas de encontro eletrônicas, videoconferências e "Computer Aided Software Engineering" (CASE).

Estas tecnologias fazem mais do que trocar senhas de acesso para comunicação. Elas criam diferentes meios de fazerem as pessoas se tornarem acessíveis umas as outras, independentemente da localização fisica. Assim, é possível criar novos ambientes de trabalho entre pessoas geograficamente distribuídas.
Estas tecnologias de conexão entre pessoas e tecnologias de acesso a informações; idéias sobre grupo e comunidade dinâmicos são úteis para explicar seus usos e entender seus potenciais.

Muito do interesse e da euforia acerca do CSCW concentra-se na variedade de disciplinas que envolve e na sua diversidade de aplicações. Entretanto, para apresentar uma visão coerente deste escopo multifuncional, é interessante enumerar os componentes do CSCW em duas categorias: os componentes tecnológicos e os humanos (WILSON, 1991). Esta separação é puramente um meio artificial para propósitos de explicação; na prática, é a estreita interconexão entre os componentes destes dois aspectos que fazem do CSCW um ramo de pesquisa multidisciplinar único e valioso.

Portanto, uma implementação bem sucedida de sistemas CSCW demanda não somente que ambos componentes tecnológicos e os humanos sejam considerados, mas também que estes sejam considerados de uma forma integrada. 


\section{Aplicações de Telecooperação}

Considerando a definição de integração, "a informação certa, na hora e lugar certo" (Bremer et al.,1997), sistemas de telecooperação constituem uma razoável alternativa para integração de aplicações, já que durante a conferência, o compartilhamento de componentes permite a uma unidade de negócio obter a informação requisitada de uma aplicação externa, sem necessidade de tê-la internamente. Apenas um sistema de compartilhamento (dados ou aplicação) é necessário entre as unidades. No caso de necessidade de dados após a seção, isto pode ser realizado pela transferência de dados.

Os compartilhamentos de aplicação e de dados são os mais poderosos componentes para possibilitar, entre os participantes de uma seção de videoconferência, uma alta interação no trabalho sobre um mesmo objeto de informação. Deste modo, eles são os mais apropriados para a integração de atividades em grupo, uma vez que os sistemas de compartilhamento permitem a um usuário acesso e visão dos dados e aplicação de um outro usuário. Um exemplo que pode ser apresentado é o fator primordial no contexto da manufatura, onde representantes de diferentes parceiros de uma cadeia de valor necessitam trabalhar em conjunto sob um mesmo desenho de uma peça.

Com estas características, os sistemas de telecooperação podem ser aplicados na integração de processos distribuídos, na cooperação entre empresas diferentes ou na conexão de cadeia de fornecedores de um setor de negócio (Eversheim et al., 1997). Ou seja, devem ser aplicados, dentro do ambiente de manufatura, em processos com comunicação intensiva, como no processo de marketing, vendas, processos de melhoria da qualidade e no processo de desenvolvimento de produto.

Alguns critérios, entretanto, devem ser considerados na escolha dos sistemas de telecooperação necessários a cada atividade, mapeada no processo específico a ser implantado o sistema. Critérios como frequência de comunicação, número de participantes, distância e pressão de tempo imposta pelo processo em questão
(Lipnack \& Stamps, 1997). A complexidade da atividade tem também uma influência na quantidade de interação necessária. Considerando estes critérios, é possível escolher adequadamente os componentes dos sistemas de telecooperação para cada atividade. Porém para que os mesmos funcionem eficientemente, faz-se necessário a estruturação de uma equipe de trabalho condizente com as exigências do trabalho em grupo à distância.

\section{Estrutura de Equipes de Trabalho a Distância}

As tecnologias de informação estão estendendo as capacidades dos trabalhadores, porém o processo de organização para realizar tarefas em grupo é ainda um trabalho humano (Lipnack \& Stamps, 1997). Portanto, se torna essencial focalizar o papel das pessoas no relacionamento organização/tecnologia no contexto de trabalho a distância.

Equipes de trabalhos a distância podem ser definidas como pequenos grupos de pessoas que trabalham ultrapassando barreiras organizacionais, suportados pelas novas tecnologias de informação. São eles os responsáveis pelo funcionamento e pelo sucesso das estruturas cooperativas, os quais enfrentam os desafios do trabalho a distância e do ambiente computacional cooperativo, mas também são aqueles que se beneficiam das vantagens deste tipo de organização.

Segundo Lipnack \& Stamps (1997), torna-se inegável que para as equipes de trabalho a distância é muito mais difícil obter sucesso do que para os tradicionais equipes "face a face". Tudo que dê errado para um grupo convencional de trabalho também dará errado para esse tipo de equipe, freqüentemente com pior intensidade. Egos, jogos de poder, pobre autoestima, sentimentos e opiniões contrariadas, ausência de liderança e falta de confiança, por exemplo, contribuem todos para o enfraquecimento das equipes. Ou seja, quando a comunicação não está sendo mais eficiente, é necessário que as pessoas tomem providências para recuperá-la. Isto ocorre pela simples razão que é muito mais difícil se comunicar através de distâncias e entre as organizações. 
PRODUÇÃO

Alguns aspectos devem ser levados em consideração tam de coaching (treinamento) (1) e orientação (Gonçalnesta nova forma de trabalho cooperativo para viabilizar seu sucesso. Um sumário destes aspectos é apresentado a seguir, com o objetivo não só de alertar sobre os desafios a serem transpostos pelas equipes de trabalho a distância, mas também mostrar algumas soluções e reações já verificadas em casos práticos, focando nas vantagens oferecidas por este novo tipo de trabalho:

- capacidade de aprender com suas próprias experiências;

Não só a organização deve aprender com sua própria experiência, mas na sua base de funcionamento, seus grupos de trabalho também devem estar preparados a reagir a mudanças inesperadas do mercado, de acordo com suas experiências anteriores, visto que ainda não existem manuais de conduta para este novo tipo de organização - Learning Systems (Marx, 1997).

- estabelecer relacionamentos de confiança;

Sem confiança mútua entre e dentro das equipes, é impossível a realização de uma tarefa. Afinal, confiança é uma condição indispensável para a otimização deste sistema de cooperação (Lipnack \& Stamps, 1997).

- estabelecimento claro das funções dos indivíduos nas equipes e a visão geral do negócio;

Sem este entendimento e senso de propósito, as equipes não alcançam os resultados que poderiam alcançar na melhoria dos resultados do negócio (Simons, 1999).

· papel da supervisão e gerência;

Os participantes de equipes virtuais não precisam de forma alguma e muito menos aceitam as formas tradicionais de supervisão e gerência. Pelo contrário, eles necessives, 1997). Os gerentes devem avaliar as equipes por base de suas performances e pela qualidade de seus resultados.

- diferenças culturais e barreiras de distância e tempo;

O desafio de integrar essas diferenças nem sempre é tão difícil e complicado quanto se pensa ou imagina. Muitas vezes equipes situadas em um mesmo ambiente de trabalho tem mais dificuldades de lidar com essa "miscelânea" de hábitos e costumes do que equipes de trabalho a distância. Dados mostram que as pessoas de uma dessas equipes que sabem que elas estão trabalhando à distância, tem mais consciência da necessidade de serem mais explícitas e claras nas suas comunicações. Enquanto, que em equipes locadas em um mesmo lugar, elas não são conscientes destas barreiras e, logo, não criam apropriadas normas compensatórias (Kraut \& Egido, 1988).

- tecnologias de suporte e;

Para viabilizar este tipo de trabalho cooperativo a distância é necessária uma infra-estrutura de comunicação, que suporte todo os tipos de tarefas e interações necessários para a realização do trabalho e da integração das equipes. Conforme discutido anteriormente, existem muitas soluções tecnológicas já existentes para este ambiente, entretanto, c que não se pode deixar de considerar é que os indivíduos não alterarão suas práticas de trabalho para se adaptarem a estas ferramentas (Araújo et al., 1995). Deste modo, estas tecnologias precisam ser usadas para suportar e aperfeiçoar o trabalho cooperativo e não substituir o antigo modo. Neste contexto, uma série de providências são imprescindiveis por parte das empresas desenvolvedoras das soluções, tais como (SIMONS, 1999): prover treinamento e adaptação, projetar interfaces amigáveis homem - máquina, estudar os impactos deste tipo de interação, etc. 
- aproveitar as vantagens do trabalho local.

Segundo alguns casos práticos (Olson \& Teasley, 1996), por melhor que seja o ambiente de interação dos grupos é necessário o relacionamento face a face pelo menos em algumas ocasiões, seja para se firmar um relacionamento de confiança, seja simplesmente para se conhecer fisicamente com quem se trabalha. Ou seja, por trás de toda esta rede tecnológica sempre vai existir um ser humano, com seus sentimentos de curiosidade, entendimento, humor e respeito.

Todos estes aspectos a serem considerados no trabalho de equipes à distância, demandam novos requisitos e habilidades ao empregado. Transformar esta força de trabalho de acordo com estas necessidades, passou a ser, então, um dos maiores desafios das empresas modernas que pretendem obter sucesso através das redes de cooperação (Gonçalves, 1997). E, como a transformação das pessoas dá-se através da educação, o desafio é principalmente de educação, reeducação e treinamento de todos os níveis e grupos dentro das empresas.

O perfil de um empregado apto a participar de equipes de trabalho a distância e contribuir para o sucesso de uma estrutura cooperativa que esteja trabalhando é resumido a seguir (Gonçalves, 1997; Kugelmass, 1996; Lipnack \& Stamps, 1997 e Picot et al., 1997):

- rápida adaptação a um ambiente de mudanças constantes. Isto é, flexibilidade na capacidade de desempenhar diferentes funções em equipes temporárias e lidar com regras e normas em situações diferenciadas,

- curiosidade para aprender e buscar novas soluções, e também saber como, aonde e quando aprender;

- criatividade e paciência para trabalhar num ambiente cooperativo a distância, consciência, confiança e respeito das barreiras a serem rompidas;
· motivação, iniciativa, atenção e responsabilidade para cumprir objetivos pré-determinados, assim como capacidade para solução de problemas e tomadas de decisão e;

- capacidade de assimilar a utilização eficiente das tecnologias de suporte ao trabalho cooperativo e de trabalhar em equipe, com competência de interação social.

Estas características nem sempre são inerentes a uma determinada pessoa. Muitas delas vão ser adquiridas com as demandas impostas por este tipo de trabalho na própria organização que deve, acima de tudo, prover meios para que seus funcionários possam se adaptar a estes requisitos. Quando se trata de adaptação, a análise da questão cultural constitui um fator primordial, já que a cooperação nem sempre se dará entre organizações que compartilham a mesma cultura.

As diferenças culturais podem provocar profundos desentendimentos entre os parceiros de uma cooperação (Metes et al., 1999). Análises como hierarquia, tempo, formas de trabalho, informação, comunicação e conflito devem ser consideradas para articular uma cultura cooperativa que considere diferentes organizações e culturas nacionais.

\subsection{Casos Práticos de Equipes de Trabalho a Distância}

A seguir descreve-se dois casos práticos de equipes trabalhando a distância no ambiente de manufatura. Os dois exemplos apresentados utilizam uma nova forma de organização a fim de adquirir agilidade no competitivo ambiente atual: a Empresa Virtual (EV).

Empresas Virtuais são baseadas principalmente na integração de competências entre diferentes empresas, provendo um produto ou serviço, que não poderia ter sido desenvolvido em termos de custo e tempo por nenhuma destas empresas sozinha. As empresas virtuais são formadas, geralmente, dentro de Organizações Virtuais 
PRODUÇÃO

(OV), que constituem redes estáveis de possiveis parceiros

A criação da VIRPLAS representa uma enorme para a formação de uma Empresa Virtual (Bremer et al., 1999). Entretanto, para o funcionamento deste novo tipo de organização faz-se necessário a utilização da metodologia de trabalho em grupo à distância, muitas vezes suportado por ferramentas computacionais.

\section{Caso Mexicano}

O projeto VIRPLAS foi criado em 1999 para constatar como o conceito de Empresa Virtual poderia explorar o desenvolvimento regional do setor indsutrial de plásticos de Monterrey - México (Molina \& Flôres, 2000). O projeto conta hoje com seis membros agregando diferentes competências da indústria plástica, tais como design de moldes e produtos, injeção plástica de componentes e comercialização de produtos plásticos e maquinário. Todas estas empresas são caracterizadas como pequenas e médias de alta tecnologia e com grande interesse e necesidade de ingressar no mercado de negócios global.

Um dos principais pontos observados no desenvolvimento deste projeto foi o interesse e o comprometimentos de todos os membros em participar desta idéia inovadora. É possível afirmar que para se ter sucesso em um projeto deste tipo, faz-se necessário que os membros desejem participar e cooperar entre si, matendo sempre uma posição pró-ativa para obter uma situação vençavença.

Outro aspecto constatado foi o esforço especial dado para melhorar as infra-estruturas físicas e de informação das empresas na criação de empresas virtuais, já que alguns membros não contavam com equipamentos de informática avançados e, os que já possuíam, não o utilizavam eficientemente. Ou seja, é importante desenvolver uma profunda avaliação da infra-estrutura cultural a fim de conscientizar os membros da VIRPLAS sobre os novos requisitos de trabalho em equipe à distância. Por exemplo, muitos membros tinham conexão de Internet, porém nem todos checavam seus e-mails diariamente.

oportunidade para as pequenas e médias empresas do setor plástico de Monterrey, levando em consideração sua proximidade com os Estados Unidos e as possibilidades de um amplo mercado. Neste aspecto, a cultura dos profissionais de Monterrey, acostumados com o relacionamento com profissionais americanos, também auxilia em possíveis cooperações.

\section{Caso Brasileiro}

O projeto VIRTEC começou em 1998 no NUMA (Núcleo de Manufatura Avançada) dentro da Escola de Engenharia de São Carlos - Universidade de São Paulo, como uma pesquisa de uma Organização Virtual Brasileira. Atualmente, o projeto conta com nove pequenas e médias empresas de alta tecnologia da cidade de São Carlos, caracterizada pelo seu peculiar ambiente econômico e acadêmico.

Porém, apesar da capacidade de geração de idéias destas empresas de alta tecnologia, elas enfrentavam a dificuldade de ganhar espaço e projeção na competitiva economia globalizada. Procurando uma abordagem que solucionasse este problema, surgiu o projeto VIRTEC com o intuito de estruturar uma base cooperativa comum, onde os membros pudessem encontrar, dentro de um ambiente de confiança mútua, a necessária infra-estrutura para explorar novas oportunidades de negócios. Deste modo, o projeto VIRTEC criou uma Organização Virtual com estas nove pequenas empresas de alta tecnologia dos ramos metal-mecânico, eletrônico, materiais cerâmicos e poliméricos.

Devido às diferentes áreas dos membros da VIRTEC está sendo necessário prover informações para suportar os processos operacionais e gerenciais da VIRTEC bem como, para desenvolver uma cultura comum para o grupo de empresas. Este projeto gerou alguns resultados positivos e identificou alguns pontos a serem levados em consideração na implementação deste novo tipo de organização do trabalho no ambiente brasileiro. 
Pode-se afirmar que o grande propulsor dos bons resultados obtidos pelo projeto se deve ao fato de se ter um certo grau de confiança entre os membros. Esta confiança vem sendo enraizada com o tempo. A cultura aberta e participativa por parte do NUMA, com relação às empresas-membro, foi um fator extremamente positivo pois permitiu que várias sugestões fossem dadas pelos membros como tentativa de trazer melhorias ao projeto. A sinceridade na demonstração das limitações da equipe, também ampliou esta abertura.

Quanto a cultura dos empresários em termos de meios de comunicação formais e informais comenta-se que a informalidade das empresas foi um fator positivo. Constatou-se também a necessidade de se ter uma infraestrutura básica para comunicação, no caso meios simples como telefone, e-mail e intranet.

A aproximação entre a Universidade e a empresa também foi um fator, citado pelos próprios membros, como extremamente positivo. Isto foi capaz de ampliar o conhecimento das empresas com relação a processo, produtos e tecnologias. Algumas outras medidas tais como, a instituição de estagiários dentro de cada empresamembro firmando mais rapidamente a parceria da universidade e empresa, através de seus alunos e as EVs criadas confirmam os bons resultados obtidos.

Entre as dificuldades apresentadas destaca-se a alteração no cronograma das atividades da VIRTEC que foram alteradas devido à natureza do trabalho dos proprietários das empresas membro os quais, freqüentemente, concentram em si próprios a maioria dos processos gerenciais e de criação. Outro ponto foi que a idéia do projeto ficou centralizada somente nos membros que participavam da $\mathrm{OV}$ e não foi disseminada para toda a empresa.

\section{Considerações Finais}

As soluções de telecooperação estão ficando mais acessíveis e estão sendo cada vez mais incorporadas ao cotidiano dos negócios. Entretanto, a análise destas soluções mostra que cuidados especiais na sua implantação e utilização, devem ser primordialmente considerados. Haja vista que muitas são as barreiras a serem transpostas pelo trabalho cooperativo a distância suportado por computador, tanto do ponto de visto humano como do tecnológico, conforme relatado. Muitos, porém, são também os benefícios proporcionados por estas tecnologias habilitadoras.

Vale ressaltar que iniciativas destinadas a aumentar a competitividade das empresas de manufatura estão transformando o processo de trabalho; entretanto, o foco reside em reeducar as pessoas que trabalham nestas organizações, para conseguir um conjunto capaz de desempenho maior. Não só os empregados vão ter de desenvolver aptidões específicas para o ambiente de cooperação, mas também as empresas devem tomar algumas considerações na sua política de Recursos Humanos para viabilizar esta evolução. Ou seja, as novas formas de organização que estão surgindo também exigem o desenvolvimento de novos modelos de administração.

Neste contexto, o estudo da cultura constitui uma das principais chaves para a criação deste ambiente cooperativo. A identificação de possíveis barreiras, principalmente no que diz respeito ao nível de confiança e cooperação entre seus membros fará com que o gerenciamento de conflitos na rede possa ser amenizado e a cultura da confiança ser estabelecida. Promovendo, então, o estabelecimento de condições básicas para esta nova forma de organização cooperativa. O estabelecimento desta confiança é um processo gradativo e tem sido alcançado com sucesso dentro do projeto VIRTEC, apesar de todas as dificuldades vivenciadas.

\section{Nota:}

1 - A palavra coaching se refere à atuação do líder dos grupos, análoga à do treinador técnico de um time de futebol (coach), quando ele prepara os jogadores, define suas posições de jogo, escolhe a estratégia de atuação e acompanha o resultado do jogo. 


\section{Referências Bibliográficas}

ARAÚJO, R.; BORGES, M.; CAMPOS, M.; CAVALCANTI, M. CSCW, Groupware e Internet Tecnologias de Suporte. http://www.cos.ufrj.br/ renata/ cscw, 1995 (15 Ago. 97).

BREMER, C.; CORRÊA, G.; RENTES, A.; ROZENFELD, R. Integrated Business Process Modelling, Simulation and Workflow Management within an Enterprise Integration Methodology. ICEIMT, 1997.

BREMER, C.; MUNDIM, A.; ORTEGA, L.; SIQUEIRA, J. ; MICHILINI, F. A Brazilian Case of VE Coordination. Capítulo do livro: Infrastructures for Virtual Enterprises - Networking Industrial Enterprises, Editado por Luis M. Camarinha-Matos and Hamideh Afsarmanesh. Lisboa/Portugal, 1999.

ELLIS, C. A .; GIBBS, S. J.; REIN, G.L. Groupware - Some Issues and Experiences. Communications of the ACM, Vol.34 - Jan. 1991.

EVERSHEIM, W.; KLOCKE, F.; PFEIFER, T.; WECK, M. Manufacturing Execellence in Global Markets. Chapman \& Hall, 1997.

MOLINA A.; FLORÊS M. Virtual Industry Clusters: Foundation to create Virtual Enterprises, accepted to be presented at BASYS'2000, September, Berlin, Germany, 2000.

GONÇALVES, J. E. L. Os Novos Desafios da Empresa do Futuro. ERA, Revista de Administração de Empresas, v.37, n.3, Jul./Set, p.10-19, 1997.

GRUDIN, J. Computer-Supported Cooperative Work: History and Focus. IEEE Computer, May 1994.

HAMUEL, G.; PRAHALAD, C. K. Competing for the Future. Boston, 1994.

KRAUT, R. \& EGIDO, C. Patterns of Contact and Communication in Scientific Research Collaboration. Proceedings of CSCW'88, New York, 1988.
KUGELMASS, J. Teletrabalho: Novas Oportunidades para o Trabalho Flexível. Atlas, 1996.

LIPNACK, J. \& STAMPS, J. Virtual Teams Reaching across Space, Time and Organizations with Technology. John Wiley \& Sons, Inc., 1997.

MARX, R. Trabalho em Grupos e Autonomia como Instrumentos da Competição. São Paulo, Atlas, 1997.

METES, G.; GUNDRY, J.; BRADISH, P. Agile networking - Competing Through the Internet and Intranet. Prentice Hall PTR. 1999.

MUNDIM, A. P. F. Proposta de um Ambiente Cooperativo Suportado por Computador para a Participação de Pequenas e Médias Empresas em Organizações Virtuais. Dissertação Mestrado em Eng, Produção, EESC - USP, 1999.

OLSON, J.; TEASLEY, S. Groupware in the Wild: Lessons Learned from a Year of Virtual Collocation. Proceedings of CSCW'96, Cambridge, 1996.

PREISS, K.; GOLDMAN, S. \& NAGER, R. Cooperate to Compete: Building Agile Business Relationships. Van Nostrand Reinhold, 1996.

PICOT, A.; REICHWALD, R. \& WIGAND, R. Information, Organisation and Management: Expanding Markets and Corporate Bondaries. John Wiles \& Sons, 1997.

SIMONS, G. A Barrier Analysis of 30 Cultural Factors Affecting Distributed Workplaces. VoNet Newsleter, Vol.2, No. 4. Http://www.virtualorganization.net, 1999 (8 Fev. 99).

WILSON, P. CSCW: An Introduction. Intellect Publishers, Oxford, UK, 1991.

WOOD, C.. Principles of Secure Information Systems Design with Groupware Examples. Computers \& Security, 12, 1993. 